\title{
Estado de situación del proceso de transición- transferencia de adolescentes con enfermedades crónicas en un hospital pediátrico de referencia nacional en Argentina \\ Status of the transition/transfer process for adolescents with chronic diseases at a national pediatric referral hospital in Argentina
}

Dra. Florencia González ${ }^{a, b}$, Dra. María de las Mercedes Rodríguez Celin ${ }^{a}$, Dra. Mariana Roizen ${ }^{b}$, Dr. Roberto Mato ${ }^{c}$ Dra. Patricia García Arrigoni ${ }^{d}$, Lic. Florencia Ugo ${ }^{e}$, Dra. Raquel Staciuk ${ }^{b}$ y Dra. Virginia Fano ${ }^{a}$

\section{RESUMEN}

Introducción: El pasaje de adolescentes de un centro pediátrico a otro de adultos es un proceso complejo. El objetivo fue evaluar el proceso de transición-transferencia de adolescentes con enfermedades crónicas en el Hospital Garrahan. Métodos: Estudio observacional, transversal, cualicuantitativo.Seobtuvieron datosestadísticos retrospectivos sobre la consulta ambulatoria de pacientes de 16-26 años y se realizaron encuestas y/o entrevistas a profesionales, adolescentes y familiares de diferentes programas de seguimiento.

Resultados: La prevalencia de atención a mayores de 16 años fue $7,2 \%$. Se encuestaron 54 profesionales asistentes, 150 pacientes (1626,7 años) y 141 familiares. Se entrevistó a 45 profesionales con cargos degestión. Profesionales: el 39\% recibió capacitación en transición. Todos identificaron obstáculos y facilitadores en los diferentes actores e instituciones intervinientes. Reconocieron la importancia en fomentar la autonomía en sus pacientes, pero solo $30 \%$ los entrevistaba solos y $56,6 \%$ les entregaba informes médicos. Estrategias: la mediana de edad de transferencia fue18 años (13-20); 62\% tenía un protocolo; $84 \%$, un acuerdo informal con otra institución; atención conjunta o paralela: $49 \%$; solo $20 \%$ utilizaba un plan de transición. Pacientes y familiares: 4,7\% de los adolescentes concurrían solos a las consultas y el profesional le había preguntado al 45\% sobre su autonomía y preparación para cuidar su salud. Los adolescentes y sus padres percibían sensaciones asociadas al proceso (con predominio, negativas) e identificaban estrategias facilitadoras, como contar con un resumen, conocer el nuevo lugar y profesionales formados.

Conclusiones: El proceso de transición del adolescente con enfermedad crónica es aún deficitario y su abordaje incluye a los equipos de salud y a las familias. Se identificaron falta de acuerdos interinstitucionales formales, aunque sí mayores acuerdos informales entre los profesionales, y la necesidad de fomentar la autonomía del paciente crónico. Entre las estrategias facilitadoras, los pacientes y sus padres reconocieron, principalmente, la necesidad de contar con un resumen médico, pautas de cuidado y confianza en el nuevo profesional.

Palabras clave: transición a la atención de adultos, adolescente, enfermedad crónica, discapacidad, determinación de las necesidades de atención médica.

http: / / dx.doi.org/10.5546/ aap.2017.562

Texto completo en inglés:

http:/ / dx.doi.org/10.5546/ aap.2017.eng.562

Cómo citar: González F, Rodríguez Celin MM, Roizen M, et al. Estado de situación del proceso de transición-transferencia de adolescentes con enfermedades crónicas en un hospital pediátrico de referencia nacional en Argentina. Arch Argent Pediatr 2017;115(6):562-569.

\section{INTRODUCCIÓN}

Los avances en la medicina en las últimas décadas han permitido que gran cantidad de niños con enfermedades pediátricas complejas sobrevivieran y llegaran a la adultez. ${ }^{1-3}$

El pasaje de adolescentes de un centro asistencial pediátrico a otro de adultos es un proceso complejo que puede conllevar dificultades para todos los actores e instituciones intervinientes. ${ }^{4-6}$

El evento puntual de pasaje es denominado transferencia. En los últimos años, ha comenzado a pensarse como un proceso más amplio y complejo denominado transición, que incluye la transferencia propiamente dicha. ${ }^{5}$

Dicho proceso requiere que los adolescentes adquieran habilidades que incrementen su autonomía para volverse individuos más responsables 
y capacitados para afrontar el autocuidado y que sus familias y el equipo de salud estén preparados para acompañarlos. ${ }^{7,8}$

En la Argentina, las estrategias que se utilizan en la práctica aún tienen un carácter asistemático y heterogéneo. ${ }^{5}$

El Hospital Garrahan es el hospital pediátrico de referencia en Argentina, donde se atienden niños y adolescentes con enfermedades crónicas y complejas. A pesar de que la reglamentación del Hospital indica que no pueden seguir atendiéndose pacientes mayores de 18 años ni ingresar nuevos mayores de 16 años, por la complejidad del problema en la práctica, esta norma no se cumple estrictamente y más del $30 \%$ de los pacientes internados son adolescentes. ${ }^{9}$

Relevamientos parciales previos mostraron que el problema se presentaba de manera muy heterogénea en los diferentes equipos de seguimiento, tanto en las estrategias utilizadas como en la importancia otorgada al tema de la transferencia. ${ }^{4,9}$

\section{OBJETIVO}

Evaluar el proceso actual de transicióntransferencia de adolescentes con enfermedades crónicas en el Hospital Garrahan.

\section{MATERIAL Y MÉTODOS}

Estudio observacional y transversal, cualicuantitativo. Se utilizaron datos prospectivos y retrospectivos.

Este estudio se realizó en el Hospital Garrahan, entre julio de 2014 y junio de 2015. Se incluyeron profesionales del equipo de salud y pacientes atendidos en el Hospital, junto con sus padres.

Para conocer la prevalencia de pacientes con patologías crónicas en seguimiento ambulatorio, se incluyeron datos estadísticos de pacientes de 16 años o mayores que habían consultado durante el año previo a la investigación (del 1-7-2013 al 30-6-2014).

Se incluyó un grupo de pacientes de 16-26 años con enfermedades crónicas y sus acompañantes adultos para identificar facilitadores y barreras en el proceso de transición. Fueron contactados al concurrir a control médico en alguno de los siguientes servicios o equipos de seguimiento: Crecimiento y Desarrollo, Gastroenterología (Enfermedades Inflamatorias Intestinales y Hepatología), Mielomeningocele, Neumonología (Asma, Enfermedad Pulmonar Obstructiva Crónica, Fibrosis Quística del Páncreas,
Trasplante Pulmonar), Neurología (Enfermedad Neuromuscular y Epilepsia), Nutrición (Diabetes), Oncología (Tumores sólidos y de Sistema Nervioso Central), Trasplante Hepático y Trasplante de Médula Ósea. Estos pacientes, además, participaron de la validación del instrumento TRAQ 5.0, cuestionario que evaluaba la preparación del paciente para la transferencia. ${ }^{10}$

Los profesionales incluidos pertenecían a los distintos grupos de seguimiento ambulatorio de pacientes con enfermedades crónicas. Se buscó la participación tanto de los profesionales asistenciales (PA) como aquellos profesionales de gestión (PG) o responsabilidad de la organización del seguimiento para conocer mejor las diferentes experiencias y estrategias utilizadas.

Se solicitó el consentimiento informado a los pacientes y sus padres o familiares y el consentimiento verbal a los profesionales. El estudio fue aprobado por los Comités de Ética y de Docencia e Investigación.

\section{Instrumentos}

Estadística sobre atención de pacientes de 16 a 26 años Datos aportados por estadística del Hospital.

\section{Encuestas-entrevistas}

Desarrolladas en consenso dentro del equipo de investigación sobre la base de los últimos avances en la materia y conocimiento del estado de la cuestión (véase Anexos 1, 2 y 3 en formato electrónico).

- Encuesta para PA: cuestionario autoadministrado con 39 preguntas, abiertas y cerradas, sobre opiniones, conocimiento, práctica y experiencias de los profesionales en relación con transición-transferencia.

- Entrevistas semiestructuradas a PG: realizadas por dos de cuatro investigadoras (FG, MR, MRC, FU) en lugar y horario convenido con el entrevistado, que tomaron registro escrito del encuentro. Se indagó sobre estrategia o protocolo y edad de transferencia, acuerdos interinstitucionales, utilización de resumen de historia clínica y/o nota de derivación o dirigida, y superposición de la atención en los dos hospitales y/o contacto posterior con los pacientes y/o médicos.

- Cuestionario para pacientes y padres: incluía 15 preguntas, abiertas y cerradas, para el adolescente y 18 para sus padres. Indagaba sobre la atención de los adolescentes y las opiniones y expectativas en relación con el proceso. 


\section{Definición de variables}

Se construyeron de novo 2 variables cuyas definiciones fueron consensuadas sobre la base de la bibliografía ${ }^{4}$ y el material emergente del análisis de las entrevistas a PG:

\section{Grado de importancia asignado al tema transición-} transferencia:

- Alto: grupo de trabajo interesado y ya trabajando en el tema.

- Medio: con interés pero aún no trabajando.

- Bajo: sin manifestar interés ni trabajo actual.

\section{Tipo de transferencia:}

- Transferencia azarosa: no había un consenso desde el Servicio ni estrategias planteadas.

- Transferencia más o menos sistematizada: no había un consenso único en el Servicio en cuanto a edad, preparación de los pacientes y/o lugar de derivación, pero se contaba con herramientas no formales que utilizaban al momento de transferir.

- Transferencia sistematizada: había consenso en relación con el momento y lugar de derivación, pero no un plan de preparación previa.

- Transición: había un proyecto de transición más o menos consolidado.

\section{Análisis}

Para el análisis de prevalencia del problema y de las respuestas a las preguntas cerradas de las encuestas a pacientes, familias y profesionales, se realizó un análisis descriptivo con las medidas de resumen correspondientes.

Para las preguntas abiertas de las encuestas y entrevistas, se realizó un análisis de contenido, que incluía tanto los ejes temáticos planteados $a$ priori como los generados en los propios datos $\mathrm{y}$ de manera comprensiva y contextual. ${ }^{11}$
Para analizar el tipo de transferencia e importancia otorgada al tema, tres investigadoras (MR, MRC, FG) clasificaron a los servicios en forma independiente. En los casos en que no era unánime, se eligió la definición según la opinión de la mayoría (2 de 3 ).

\section{RESULTADOS \\ Datos estadísticos}

El grupo de pacientes de entre 16 y 26 años representaba el 7,2\% de las consultas ambulatorias (24394 sobre un total de 340597 consultas). Dentro de este grupo, el $31 \%$ fueron consultas de pacientes mayores de 18 años. No se incluyeron consultas de emergencias.

\section{Profesionales}

Se realizaron 54 encuestas a PA y 45 entrevistas a aquellos PG de los programas de atención contactados, de 36 servicios del Hospital. No hubo ninguna negativa a participar. A continuación, se presentan los hallazgos más importantes:

Conocimiento sobre transición (PA): solo un 39,6\% refirió alguna formación curricular básica o posbásica, y la mayoría (68\%) había recurrido de forma individual a la lectura relacionada.

Prácticas en la asistencia con adolescentes con enfermedades crónicas (PA): como puede verse en la Tabla 1, la mayoría refirió prácticas que, de alguna manera, fomentaban la autonomía. Sin embargo, casi un tercio nunca realizaba entrevistas a solas con los adolescentes y era variable la presentación de informes completos.

Obstáculos y facilitadores (PA, PG): los profesionales refirieron obstáculos en los diferentes actores intervinientes en el proceso (familias, pacientes, médicos, instituciones). También refirieron necesidades y facilitadores en los distintos niveles donde encontraban los obstáculos (Tablas 2 y 3).

TABLA 1. Prácticas en la atención de pacientes adolescentes autorreportadas por los profesionales del Hospital. N: 54

\begin{tabular}{|c|c|c|c|}
\hline & Siempre & A veces & Nunca \\
\hline ¿Dirige parte de las preguntas al paciente? & $66 \%$ & $34 \%$ & ---- \\
\hline ¿Mantiene a los padres en la sala de espera durante parte de la visita? & $11,3 \%$ & $58,5 \%$ & $30,2 \%$ \\
\hline ¿Tiene en cuenta el nivel de autonomía de los pacientes frente a las actividades de la vida diaria? & $83 \%$ & $15,1 \%$ & $1,9 \%$ \\
\hline ¿Introduce el tema de la preparación para comenzar un seguimiento como un adulto en la consulta? & $65,4 \%$ & $26,9 \%$ & $7,7 \%$ \\
\hline ¿Instruye al paciente sobre su patología? & $88,5 \%$ & $11,5 \%$ & ---- \\
\hline $\begin{array}{l}\text { ¿Instruye al paciente sobre los nombres de la medicación, dosis, posibles alergias, } \\
\text { efectos adversos o interacciones? }\end{array}$ & $62,7 \%$ & $33,3 \%$ & $4 \%$ \\
\hline ¿Provee al paciente de informes médicos completos? & $56,6 \%$ & $39,6 \%$ & $3,8 \%$ \\
\hline ¿Estimula al paciente a buscar o ponerse en contacto con un médico de adultos? & $57,7 \%$ & $32,7 \%$ & $9,6 \%$ \\
\hline ¿Intenta identificar distintas barreras desde el paciente o su familia para la transición/transferencia? & $50 \%$ & $40,4 \%$ & $9,6 \%$ \\
\hline
\end{tabular}


También surgieron como obstáculos el grado de discapacidad, la pobre adherencia al tratamiento y la falta de lugares adecuados para transferir en el interior del país.

Estrategias para la transición-transferencia (PG): el 62\% tenía una estrategia / protocolo para el proceso de transición-transferencia, con las siguientes características.

- Edad: el 73\% transfería a sus pacientes a una edad determinada. Todos a partir de los 16 años como mínimo, excepto 1 servicio. La mediana de edad de derivación referida fue de 18 años (rango de 13-20).

- Acuerdos: ninguno tenía acuerdo formal con las instituciones a las que transferían; el $84 \%$ tenía algún acuerdo informal, y el 16\%, ninguno. La comunicación y contacto posterior con los profesionales era muy variable (32\% establecía contacto posterior; $30 \%$ no lo hacía y $38 \%$, solo a veces).

- Atención conjunta o paralela: la mayoría reconocía la utilidad de un período de superposición en la atención entre centros. El $49 \%$ realizaba, de alguna manera, esta práctica (atención conjunta, única visita posterior o superposición por un tiempo).
- Tipo de transferencia: el $20 \%$ tenía en marcha un plan de transición; $20 \%$ realizaba una transferencia sistematizada; $40 \%$, una transferencia más o menos sistematizada, $y$, en el 20\%, era azarosa.

- Grado de importancia asignado al tema: alta en el $40 \%$, media en el $47 \%$ y baja en $13 \%$ de los servicios. El 51,9\% estaba trabajando en el tema.

Otras estrategias existentes (PG):las entrevistas permitieron conocer diferentes experiencias ya desarrolladas, muy variadas y que pudieran resultar como modelo: atención en consultorio diferenciado para adolescentes (infectología -virus de inmunodeficiencia humana, VIH-, trasplante hepático, cardiopatías congénitas complejas); talleres mensuales de formación y expresión para pacientes y pares (VIH); talleres multidisciplinarios para padres y pacientes antes de la transferencia (Equipo de enfermedades neuromusculares); evaluación de la preparación para transferencia según protocolo (Nefrología); ateneos y atención conjunta con médicos de adultos (Gastroenterología -enfermedad inflamatoria intestinal, EII-, Neumonología -fibrosis quística,

TABLA 2. Obstáculos o barreras autorreportados por los profesionales encuestados. N: 54

\begin{tabular}{|c|c|c|c|}
\hline Obstáculos & Muy frecuente & A veces & Nunca \\
\hline \multicolumn{4}{|l|}{ Desde la institución o sistema de salud } \\
\hline Pasaje determinado solo por la edad del paciente. & $49 \%$ & $39,2 \%$ & $11,8 \%$ \\
\hline Falta de comunicación, interrelación, coordinación de acciones entre centro de niños y adultos. & $61,5 \%$ & $34,6 \%$ & $3,9 \%$ \\
\hline Falta de comunicación, interrelación, coordinación de acciones entre profesionales del & & & \\
\hline hospital pediátrico para acordar las estrategias para la transición. & $44,2 \%$ & $46,2 \%$ & $9,6 \%$ \\
\hline Se practican, en general, acciones aleatorias o improvisadas que dependen de cada profesional. & $52 \%$ & $44,2 \%$ & $3,8 \%$ \\
\hline \multicolumn{4}{|l|}{ Desde los pediatras/especialistas pediátricos } \\
\hline Sobreprotección/paternalismo. & $54,7 \%$ & $39,6 \%$ & $5,7 \%$ \\
\hline Falta de información trasmitida al paciente. & $23,1 \%$ & $51,9 \%$ & $25 \%$ \\
\hline Desvalorización de los médicos de adultos. & $30,2 \%$ & $32,1 \%$ & $37,7 \%$ \\
\hline No estimulación de la autonomía. & $21,2 \%$ & $53,8 \%$ & $25 \%$ \\
\hline Falta de entrega de información necesaria para el seguimiento por profesionales de adultos. & $9,4 \%$ & $52,8 \%$ & $37,8 \%$ \\
\hline \multicolumn{4}{|l|}{ Desde los pacientes adolescentes } \\
\hline Falta de autonomía. & $33,3 \%$ & $64,7 \%$ & $2 \%$ \\
\hline Enfermedad grave o discapacidad marcada. & $40,4 \%$ & $59,6 \%$ & \\
\hline Falta de confianza en el nuevo equipo tratante. & $42,3 \%$ & $53,8 \%$ & $3,9 \%$ \\
\hline Pobre adherencia al tratamiento. & $24 \%$ & $74 \%$ & $2 \%$ \\
\hline \multicolumn{4}{|l|}{ Desde la familia } \\
\hline El pediatra o especialista pediátrico es el médico que más conoce al paciente y su familia. & $83 \%$ & $15,1 \%$ & $1,9 \%$ \\
\hline Vínculo estrecho entre el pediatra y la familia. & $88,7 \%$ & $9,4 \%$ & $1,9 \%$ \\
\hline El hospital pediátrico le resulta un ambiente familiar. & $88,7 \%$ & $11,3 \%$ & \\
\hline Resistencia por parte de la familia para la autonomía del adolescente. & $47,2 \%$ & $50,9 \%$ & $1,9 \%$ \\
\hline Miedo y ansiedad por el proceso de transición. & $69,8 \%$ & $30,2 \%$ & --- \\
\hline \multicolumn{4}{|l|}{ Desde los médicos de adultos } \\
\hline Menor experiencia en patologías crónicas de la niñez. & $60,4 \%$ & $34 \%$ & $5,6 \%$ \\
\hline Falta de hábito de incorporar a la familia en la atención. & $57,7 \%$ & $40,4 \%$ & $1,9 \%$ \\
\hline
\end{tabular}


FQ-); cursos de formación para médicos de adultos (enfermedades metabólicas, cardiopatías congénitas). Además, se llevaron adelante múltiples proyectos y becas de investigación que ampliaban el conocimiento y la experiencia (nutrición, reumatología, salud mental, servicio social, hematooncología, crecimiento y desarrollo, relaciones institucionales y adolescencia).

\section{Pacientes y sus familias}

Se incluyeron 150 pacientes, con una edad media de 17,5 años (16-26,7) y, en 141 casos, también algún familiar. Solo 2 pacientes se negaron a participar. Sus opiniones se resumen a continuación.

\section{Lugar de los adolescentes en las consultas}

Solo 4,66\%refirieron concurrir a las consultas solos; el resto lo hacía con sus padres.

Según la visión de los adolescentes, solo al $45 \%$ se le había preguntado sobre cómo se manejaban con lo referente a su salud y, para la mayoría $(73 \%)$, estas preguntas habían sido dirigidas tanto a ellos como a sus padres.
Las preguntas, generalmente, se relacionaban con su estado general, cumplimiento de los tratamientos y cuestiones personales. Muy pocos expresaron que se les había preguntado sobre pautas de autonomía o haber hablado sobre las características de la atención en un hospital de adultos $(8,66 \%$ de adolescentes y $4,96 \%$ de padres).

Sin embargo, al otorgarles opciones de respuesta en la encuesta, las dos más elegidas fueron: “Me van explicando cada vez más para que vaya entendiendo todo y hablan más directamente conmigo" $(57,5 \%)$ y "Me explicaron y tengo un resumen y los cuidados que necesito" (43,2\%). Asimismo, en relación con la comunicación con sus padres, el 51\% refirió: "Les van enseñando de a poco y todo es muy natural".

Con respecto a la opinión de los padres, también percibieron que el proceso de transición aún era deficitario: solo menos de la mitad (41\%) refirió que los médicos les habían preguntado sobre la preparación de sus hijos para manejarse solos con lo referente a su salud y solo el $51 \%$ que se les había planteado el tema de la

TABla 3. Percepciones de los obstáculos y necesidades referidas por los profesionales entrevistados

\begin{tabular}{|c|c|}
\hline Obstáculo/facilitador/necesidad & Percepción \\
\hline \multicolumn{2}{|l|}{ Obstáculo } \\
\hline Falta de comunicación entre los profesionales. & $\begin{array}{l}\text { "Muchas veces, desde la especialidad principal, lo derivan y } \\
\text { no avisan a los otros miembros del equipo". }\end{array}$ \\
\hline La sobreprotección y paternalismo sobre sus pacientes. & $\begin{array}{l}\text { "Los pediatras somos más cálidos, perceptivos, hay más } \\
\text { fraternidad". }\end{array}$ \\
\hline $\begin{array}{l}\text { La menor experiencia de los profesionales de adultos en el } \\
\text { manejo de patologías crónicas de la niñez y falta de } \\
\text { incorporación de la familia en la atención. }\end{array}$ & $\begin{array}{l}\text { "Muchos tratamientos no los conocen o no tienen } \\
\text { experiencia". }\end{array}$ \\
\hline $\begin{array}{l}\text { La estrecha relación entre las familias y } \\
\text { los profesionales pediátricos. }\end{array}$ & $\begin{array}{l}\text { "Al ser niños que se siguen hace mucho tiempo en el } \\
\text { Hospital, les cuesta el cambio, tanto a las familias como a } \\
\text { los especialistas". }\end{array}$ \\
\hline \multicolumn{2}{|l|}{ Facilitador } \\
\hline $\begin{array}{l}\text { Conformación de redes con profesionales rotantes } \\
\text { que han hecho alguna beca de formación. }\end{array}$ & $\begin{array}{l}\text { "Nos es útil contar con los nexos que generamos con } \\
\text { los profesionales rotantes y la formación que adquieren". }\end{array}$ \\
\hline $\begin{array}{l}\text { Un período de superposición en la atención de los } \\
\text { centros pediátricos y de adultos. }\end{array}$ & $\begin{array}{l}\text { "Este debe ser un proceso, por lo que debe haber una } \\
\text { instancia intermedia en la que los pacientes se atiendan en } \\
\text { los dos lados". }\end{array}$ \\
\hline $\begin{array}{l}\text { Pasaje a una instancia institucional, con acuerdos formales } \\
\text { a todo el sistema de salud. }\end{array}$ & $\begin{array}{l}\text { "Debe haber una política institucional, con comunicación } \\
\text { entre directores, que involucre al paciente, servicio social y } \\
\text { prestaciones". }\end{array}$ \\
\hline $\begin{array}{l}\text { Trabajo con los adolescentes para prepararlos y } \\
\text { fomentar suautonomía. }\end{array}$ & $\begin{array}{l}\text { "He tenido una experiencia muy fea por falta de } \\
\text { preparación, en la que, de un día para otro, les tenía que } \\
\text { decir que no se podían seguir más acá. De eso aprendí". }\end{array}$ \\
\hline $\begin{array}{l}\text { Cambios en la historia clínica y en los resúmenes } \\
\text { que se entregan. }\end{array}$ & $\begin{array}{l}\text { "Hacer algún cambio en la historia clínica electrónica, } \\
\text { por ejemplo, una alarma: paciente en plan de transición". } \\
\text { "Solo un resumen no alcanza, deberíamos darles una guía } \\
\text { con los cuidados que necesitan". }\end{array}$ \\
\hline $\begin{array}{l}\text { Garantizar a los pacientes la continuidad de la relación } \\
\text { con el equipo pediátrico. }\end{array}$ & “No rompemos el vínculo, siempre mail o directamente vienen". \\
\hline
\end{tabular}


transferencia, a pesar de ser sus hijos mayores de 16 años. Sin embargo, el 90\% refirió no encontrar dificultades desde el profesional para dirigirse a los adolescentes, un 15\% respondió que siempre habían tenido en cuenta a su hijo en las consultas, y la edad media a la que habían empezado a hacerlo era 12 años (2-16).

\section{Momento para la transferencia:}

El $44 \%$ de los pacientes creía que se encontraba en un momento adecuado para ser atendido por un médico de adultos; sus principales argumentos eran el sentirse mayores y preparados. Los motivos por los que no sabían si se encontraban preparados $(46 \%)$ o directamente creían no estarlo (10\%) fueron el acostumbramiento con la atención en el Hospital, el miedo a diferencias en el trato, la edad y la falta de preparación.

En relación con los padres, un porcentaje similar a sus hijos (39\%) creía que estos se encontraban listos para la transferencia, mientras que el porcentaje de los que respondieron que no se encontraban preparados fue mayor $(27 \%$ vs. $10 \%$ ). El resto no sabía. Las razones en relación con estar en un momento adecuado fueron similares a las de sus hijos y relacionadas con la edad: "Ya va a tener 20, tiene pudor de estar con chicos", "Ya es adulta, va a la facultad, necesita rodearse de gente adulta, sino no puede crecer".

Los motivos referidos por los cuales creían que sus hijos no estaban preparados fueron la costumbre, la edad, la falta de autonomía y de confianza en el nuevo médico: "Son muchos años acá, muchas internaciones, acá nos sentimos en casa", "Todavía no se maneja solo", "No veo profesionales formados en adolescencia y discapacidad".

\section{Sensaciones asociadas al proceso:}

Al 40\% de los adolescentes y $68,6 \%$ de los padres este proceso le causaba alguna sensación, en general, negativa y, en menor medida, positiva. En la Tabla 4, pueden leerse algunos ejemplos de cómo se sintieron al hablarles por primera vez de la transferencia. A la mayoría de los padres $(74 \%)$ le inquietaba que pudiera haber diferencias en el "trato".

\section{Estrategias:}

En relación con qué consideraban que podría ayudar en el momento de la transicióntransferencia, las opciones más elegidas por los pacientes fueron contar con un resumen de historia clínica $(59,9 \%)$ y conocer el nuevo lugar $(54,4 \%)$; mientras que, para los padres, sería que los médicos estuvieran formados en la patología de su hijo $(70,7 \%)$ y contar con un resumen de historia clínica $(70,7 \%)$.

\section{DISCUSIÓN}

El presente estudio permitió realizar un diagnóstico de situación amplio y actual sobre el proceso de transición-transferencia en los servicios ambulatorios que atendían apacientes con enfermedades crónicas del Hospital Garrahan. En forma similar al relevamiento realizado en 2007-2008, ${ }^{4}$ en varios servicios del Hospital y en otros hospitales pediátricos, se encontró amplia variabilidad con experiencias que iban desde transferencias más programadas a otras más azarosas y también en el grado de importancia otorgado. En ninguno de los 2 relevamientos, se encontraron acuerdos interinstitucionales formales. Sí se encontró una diferencia en la frecuencia de acuerdos informales: actualmente,

TABLA 4. Sensaciones referidas por los adolescentes y sus familias frente al planteo de la necesidad de transferencia a una institución de adultos

\begin{tabular}{ll}
\hline \multicolumn{1}{c}{ Sensaciones negativas } & \multicolumn{1}{c}{ Sensaciones positivas } \\
\hline Adolescentes & \\
"Miedo, no sé lo que podrá pasar". & "Normal, tranquilo, ya sabía que en algún momento pasaría". \\
"Rara, no entendía mucho". & "Alegre, más segura de mí misma". \\
"Más o menos, porque no sé manejarme sola". & "Estoy más grande, responsable, independiente". \\
& "Puede funcionar mejor". \\
\hline
\end{tabular}

Padres

"Desprotegida y preocupada".

"Te da cosa, pero es necesario, ya son grandes".

"Nervios, va a ser empezar todo de nuevo".

"Triste, porque me di cuenta de que ellos no estarán".

"Segura, porque nos prepararon mucho antes".

"Los médicos pediatras se ocupan más". 
el $84 \%$ tiene algún acuerdo informal y, en el relevamiento anterior, solo un $30 \%,{ }^{4}$ por lo que este cambio muestra un avance.

En relación con los obstáculos, en coincidencia con hallazgos previos, se encontraron en todos los distintos actores involucrados: adolescente, familia, pediatras y médicos de adultos y desde las instituciones o sistemas. Estos obstáculos fueron comparados con aquellos analizados en un consenso de la Sociedad Argentina de Pediatría, en el que se encontraron resultados similares. ${ }^{5}$ Asimismo, estas barreras ya habían sido descritas internacionalmente, con independencia del nivel de desarrollo de los países. Un ejemplo cercano es el documento elaborado por la Sociedad Chilena de Pediatría en el año 2011, en el que, aparte de mencionar dichas barreras, se sumó la transferencia a una edad fija de 15 años. ${ }^{6}$

Dentro de las estrategias sugeridas por los profesionales con cargos de gestión, surgieron como más relevantes la necesidad de acuerdos formales, formación de profesionales en el lugar de origen, formación de redes y fomentar la autonomía de los adolescentes. Desde las familias y pacientes, facilitaría el proceso contar con un resumen de historia clínica, la formación del profesional que reciba al paciente y conocer el lugar al cual es derivado. Estas estrategias coinciden con recomendaciones de la bibliografía, sobre todo, en la importancia de contar con un resumen médico actualizado y accesible. También se sugiere contar con un plan escrito de transición desde los 14 años, elaborado junto con el paciente y su familia, y actualizado periódicamente, así como utilizar guías para evaluar y trabajar las habilidades de los adolescentes. Es importante adecuar la preparación según el intervalo etario y de acuerdo conla situación de cada paciente. ${ }^{12,13,7}$

Otro punto que se encontró y que fue coincidente con la bibliografía fue la necesidad de mejorar la comunicación entre el profesional pediátrico y el de adultos y hacer más eficiente el traspaso de la información. ${ }^{6}$

Asimismo, la diferencia encontrada en relación con la edad "ideal" de transferencia (en padres, mayor que en sus hijos y, en ambos, mayor que en los profesionales) también debería tenerse presente ante la aparición de dificultades para el cambio.

Por último, algunas de las dificultades serán más fáciles de abordar por los distintos actores interesados, pero otras requerirán una estrategia más global a nivel interinstitucional.

\section{CONCLUSIONES}

El proceso de transición del adolescente con enfermedad crónica es aún deficitario y su abordaje incluye a los equipos de salud y a las familias. Se identificaron falta de acuerdos interinstitucionales formales, aunque sí mayores acuerdos informales entre los profesionales, y la necesidad de fomentar la autonomía del paciente crónico. Entre las estrategias facilitadoras, los pacientes y sus padres reconocieron, principalmente, la necesidad de contar con un resumen médico, pautas de cuidado y confianza en el nuevo profesional.

\section{Financiamiento}

La Dra. Florencia González recibió una beca de investigación otorgada por la Coordinación de Investigación y financiada por la Fundación del Hospital de Pediatría "Prof. Dr. Juan P. Garrahan" durante los años 2014 ("Construcción de un programa de transición para pacientes adolescentes-adultos jóvenes con enfermedades crónicas en el Hospital Garrahan: sentando las bases") y 2015 ("Ampliación de la validación y evaluación de la utilidad del TRAQ 5.0 y difusión de la problemática de la transición en el Hospital Garrahan").

\section{REFERENCIAS}

1. McPherson M, Arango P, Fox H, et al. A new definition of Children with Special Health Care Needs. Pediatrics 1998;102(1 pt 1):137-40.

2. Gortmarker S, Sappenfield W. Chronic childhood disorders: Prevalence and impact. Pediatr Clin North Am 1984:31(3):3-18.

3. NewacheckP, Stoddard J. Prevalence and impact of multiple childhood chronic illnesses. J Pediatr 1994;124(1):40-8.

4. Ugo F, Schejter VH, Carniglia L, et al. La transición del cuidado en el paciente crónico: El largo camino de un hospital pediátrico a un hospital de adultos. Med Infant 2009;16(1):16-23.

5. Garategaray M, Rodriguez Ponte M, Breitman F, et al. Transición del adolescente con enfermedades crónicas. Sociedad Argentina de Pediatría, 2011. [Consulta: 4 de julio de 2017]. Disponible en: http://www.sap.org.ar/ docs / profesionales/transicion_del_adolescente_con_ enfermedades_cronicas.pdf.

6. Lizama CM, Ávalos AM, Vargas CN, et al. Transición al cuidado de la vida adulta, de niños y adolescentes con necesidades especiales de atención en salud: recomendaciones del Comité NANEAS Sociedad Chilena de Pediatria. Rev Chil Pediatr 2011;82(3):238-44.

7. De Cunto CL. Transición en la atención médica, de la pediatría a la medicina del adulto. Arch Argent Pediatr 2012; 110(4):341-7.

8. Zhang LF, Ho JS, Kennedy SE. A systematic review of the psychometric properties of transition readiness assessment tools in adolescents with chronic disease. BMC Pediatr 2014;14:4. 
9. Mato R, Fernández Do Campo, Breyter $\mathrm{P}$, et al. Transición de Adolescentes con Enfermedad Crónica. Resultados de 6 meses de una beca en el Hospital Garrahan enfocada a esta temática. Poster 95 presentado en: Recorrida Poster Digital. $8^{\circ}$ Congreso Argentino de Salud Integral del Adolescente; 15-18 de mayo de 2013; Cuidad Autónoma deBuenos Aires, Argentina.

10. González F, Roizen M, Rodríguez Celin M, et al. Validación español-argentina del cuestionario de transición a la atención médica del adulto en adolescentes con enfermedades crónicas. Arch Argent Pediatr 2017;115(1):18-27.
11. Minayo MCS. El desafío del conocimiento. Investigación cualitativa en salud. Buenos Aires: Lugar; 2004.

12. American Academy of Pediatrics, American Academy of Family Physicians, American College of PhysiciansAmerican Society of Internal Medicine. A consensus statement on health care transitions for young adults with special health care needs. Pediatrics 2002;110(6 pt 2):1304-6.

13. American Academy of Pediatrics, American Academy of Family Physicians, American College of Physicians, et al. Supporting the health care transition from adolescence to adulthood in the medical home. Pediatrics 2011;128(1):182200.

"La salud es algo demasiado importante como para dejarla en manos de empresas cuyo fin principal es el lucro, porque el lucro no tiene límites."

Mario Bunge

"La tasa de crecimiento económico ya no depende de la tecnología o de las maquinarias que se posea, sino de lo que haga la gente" 


\section{ANEXO 1: ENCUESTA A MÉDICOS}

\section{Proyecto: “Construcción de un programa de transición para pacientes adolescentes-adultos jóvenes con enfermedades crónicas en el Hospital Garrahan: sentando las bases". Cuestionario para el equipo de salud}

Los avances en la medicina en las últimas décadas han favorecido el diagnóstico,tratamiento y manejo de muchas enfermedades pediátricas y, con esto, ha aumentado la sobrevida de muchísimos pacientes que llegan a la etapa adulta. Esto representa un panorama y una necesidad diferente en la atención médica.

Con este cuestionario, exploraremos el conocimiento actual y la opinión de los profesionales del Hospital sobre el tema y describiremos las estrategias existentes en los distintos servicios a la hora de transferir a los pacientes al sistema de salud de adultos, con el fin de sentar las bases para construir un programa de transición en nuestro Hospital.

Para el fin descrito, le pedimos que conteste según su opinión las siguientes preguntas y que aquellas que considere que tienen que ver más con una postura general del Servicio las discuta con su grupo de trabajo. En el momento de la devolución, le pediremos que nos cuente más en profundidad sobre las estrategias actuales utilizadas.

Nombre y apellido:

Fecha:

Cargo:

Edad:

Sector al que pertenece:

Programa de atención al que pertenece:

\section{Conocimiento:}

1. En su formación curricular, básica, posbásicao a través del tiempo en educación continua (en los diversos ámbitos, espacios, etc.), ¿estuvo contemplado el tema de la transición y transferencia? Sí (1). $\square-$ No (0). $\square \quad$ ¿De qué manera?

2. ¿Alguna vez recurrió a la lectura de diferente información para conocer más sobre este tema? Sí (1). $\square-$ No (0). $\square \quad$ ¿Recuerda dónde?

\section{Práctica:}

3. ¿Tienen alguna estrategia/ protocolo como Servicio para la transferencia de pacientes a hospital de adultos? Sí (1). 口- No (0).

4. En su Servicio, ¿transfieren a los pacientes a un especialista/hospital de adultos a una edad determinada? Sí (1). $\square-$ No (0). $\square \quad$ Edad:

5. ¿Tienen conocimiento de qué sucede luego con estos adolescentes? Sí (1). $\square-$ No (0). $\square$ A veces (2). $\square$ ¿Siguen en contacto con el paciente? Sí (1). $\square-$ No (0). $\square$ A veces (2).

¿Siguen en contacto con los nuevos profesionales? Sí (1). $\square-$ No (0). $\square$ A veces (2). $\square$

La atención de adolescentes con enfermedades crónicas es un desafío, a veces, difícil de manejar, sobre todo, cuando seguimos a nuestros pacientes desde niños. Si usted piensa en su práctica cotidiana, ¿con qué frecuencia cree que realiza las siguientes prácticas cuando está atendiendo a adolescentes?

Siempre (1) A veces (2) Nunca (3)

6. ¿Dirige parte de las preguntas al paciente?

7. ¿Mantiene a los padres en la sala de espera durante parte de la visita?

8. ¿Tiene en cuenta el nivel de autonomía de los pacientes frente a las actividades de la vida diaria?

9. ¿Introduce el tema de la preparación para comenzar un seguimiento como un adulto en la consulta?

10. ¿Instruye al paciente sobre su patología?

11. ¿Instruye al paciente sobre los nombres de su medicación, dosis, posibles alergias, efectos adversos o interacciones?

12. ¿Provee al paciente de informes médicos completos?

13. ¿Estimula al paciente a buscar o ponerse en contacto con un médico de adultos?

14. ¿Intenta identificar distintas barreras desde el paciente o su familia para la transición/transferencia? 


\section{Opiniones:}

15. ¿A qué edad le parecería a usted que sería óptimo transferir a los pacientes?

16. Además de la edad, ¿qué otras variables le parece que tendrían que tenerse en cuenta para la transferencia?

Los obstáculos que se enumeran en la tabla siguiente han sido presentados como posibles en un documento colaborativo de la Sociedad Argentina de Pediatría (SAP). ${ }^{1}$ A la hora de transferir a los pacientes adolescentes, teniendo en cuenta su experiencia profesional, indique con qué frecuencia se encuentra con los siguientes obstáculos:

Obstáculos

Muy frecuente (1) A veces (2) Nunca (3)

Desde la institución o sistema de salud

17. Pasaje determinado solo por la edad del paciente.

18. Falta de comunicación, interrelación, coordinación de acciones entre centro de niños y adultos.

19. Falta de comunicación, interrelación, coordinación de acciones entre profesionales del

hospital pediátrico para acordar las estrategias para la transición.

20. Se practican, en general, acciones aleatorias o improvisadas que dependen de cada profesional.

Desde los pediatras/especialistas pediátricos

21. Sobreprotección/paternalismo.

22. Falta de información trasmitida al paciente.

23. Desvalorización de los médicos de adultos.

24. No estimulación de la autonomía.

25. Falta de entrega de información necesaria para el seguimiento por profesionales de adultos.

Desde los pacientes adolescentes

26. Falta de autonomía.

27. Enfermedad grave o discapacidad marcada.

28. Falta de confianza en el nuevo equipo tratante.

29. Pobre adherencia al tratamiento.

\section{Desde la familia}

30. El pediatra o especialista pediátrico es el médico que más conoce al paciente y su familia.

31. Vínculo estrecho entre el pediatra y la familia.

32. El hospital pediátrico le resulta un ambiente familiar.

33. Resistencia por parte de la familia para la autonomía del adolescente.

34. Miedo y ansiedad por el proceso de transición.

Desde los médicos de adultos

35. Menor experiencia en patologías crónicas de la niñez.

36. Falta de hábito de incorporar a la familia en la atención.

37. ¿En su práctica habitual usted encuentra otros obstáculos?

\section{Para terminar...}

38. ¿Algún integrante de su Servicio trabaja actualmente en estrategias para la transición-transferencia de pacientes?

Sí (1). $\square-$ No (0). $\square$ ¿Quién?

39. ¿A algún integrante del Servicio le interesaría formar un equipo interdisciplinario para abordar el tema de la transición-transferencia en el Hospital?

Sí (1). $\square-$ No (0). $\square$ ¿A quién?

\footnotetext{
${ }^{7}$ http: / / www.sap.org.ar/docs/profesionales/transicion_del_adolescente_con_enfermedades_cronicas.pdf.
} 


\section{ANEXO 2: ENCUESTA A PACIENTES}

Las siguientes preguntas nos sirven a nosotros para saber qué opinas vos y los chicos que están en una situación parecida a la tuya sobre lo que estamos haciendo y lo que necesitamos cambiar. Como las respuestas son personales y anónimas, te pedimos que seas sincero; no hay respuestas correctas o incorrectas. Cualquier cosa, preguntanos.

1. ¿Los médicos suelen hacer preguntas sobre cómo te vas preparando para manejarte solo con lo referente a tu salud?

No(0). $\square \quad$ A veces(1). $\square \quad$ Casi siempre(2). $\square$

2. ¿A quién le hacen las preguntas?

No preguntan(0). $\square$ A mí(1). $\square$ A mis padres(2). $\square \quad$ A ambos(3). $\square$

3. Cuando preguntan, ¿sobre qué preguntan?

4. ¿Cómo concurrís a las consultas con los médicos?

Solo(1). $\square$ Acompañado pormis padres(2). $\square$ Acompañado por otra persona (3). $\square$

5. ¿Creés que estás en un momento adecuado para ser atendido por un médico de adultos? No(0). $\quad$ Sí(1). $\square$ No sé(2). ¿ ¿Por qué?

6. ¿Cuál creés que sería la edad ideal para pasar a un servicio de adultos?

7. ¿Cómo te imaginás que va a ser la derivación? ¿En qué momento?

8. ¿Ese momento te genera alguna sensación?

No(0). $\square \quad$ Sí(1). ¿Cuál?

9. ¿Qué cosas creés que te podrían ayudar? (Podés responder más de una opción).

- Conocer el lugar(1).

- Que esté cerca de mi casa(2).

- Contar con un resumen de mi historia clínica (3). $\square$

- Que me conozcan cuando llegue(4).

- Médicos formados en mi patología (5).

- Vía de comunicación con el hospital (6). $\square$

10. ¿Cómo sentís que es la actitud de tus médicos con respecto a que vayas aprendiendo sobre tu salud?

- Siguen hablando con mis padres, a mí todavía no me explican lo que me pasó, pasa o me puede pasar(1).

- Me van explicando de a poco lo que me va pasando, pero no lo que es mi enfermedad en sí o lo que me pasó antes(2)..

- Me van explicando cada vez más para que vaya entendiendo todo lo que respecta a mi salud y hablan más directamente conmigo(3).

- Me explicaron y tengo un resumen sobre mi historia y los cuidados que necesito(4). 
11. Con respecto a tus padres, ¿cómo es la relación con respecto a lo que sucede con tu salud? (Podés responder más de una opción).

- Me sobreprotegen y no dejan que me ocupe de nada(1).

- Me van enseñando de a poco mis cuidados, pero les da miedo/les cuesta(2). $\square$

- Me van enseñando de a poco y todo es muy natural(3)..

- Me dejan solo(4). .

12. ¿Desde qué edad te dejan hacer aunque sea algo solo con lo que respecta a tu salud? Aún no(0). $\square$ Desde los años.

13. ¿Ya se planteó el pasaje a un hospital de adultos?

No(0). $\square \quad$ Sí(1). $\square$ Si ya se planteó:

¿Cómo te sentiste cuando te hablaron por primera vez?

14. ¿Cómo te ves en 5 años?

15. ¿Y en 10 años?

16. ¿Querés decir algo más? 


\section{ANEXO 3: ENCUESTA A PADRES}

Además de conocer la opinión de su hijo sobre el pasaje a la medicina del adulto y el nivel de preparación para él, nos interesa conocer algunas opiniones de los padres o cuidadores sobre este tema, que son quienes se han ocupado de su cuidado por mucho tiempo y quienes los acompañarán en este proceso. Queremos pedirle, entonces, que piense cómo fue hasta acá la atención de su hijo en el hospital y que nos responda las siguientes preguntas:

1. La enfermedad crónica de su hijo, ¿cree que tiene repercusiones en la dinámica familiar? No(0). $\square \quad$ Sí(1). $\square$ ¿En qué le parece?

2. ¿Hay algún otro miembro de la familia con la misma enfermedad? No(0). $\square \quad$ Sí(1). ¿Quién?

3. ¿Cómo es su relación con respecto a lo que sucede con la salud e independencia de su hijo? (Puede responder más de una opción).

- Lo sobreprotegen y no dejan que se ocupe de nada(1). $\square$

- Le van enseñando de a poco sus cuidados, pero les da miedo/les cuesta(2). .

- Le van enseñando de a poco y todo es muy natural(3)..

- Lo dejan solo(4)..

4. ¿Dentro del equipo de profesionales que atiende a su hijo hay algún médico referente o que funcione como médico de cabecera?

No(0). $\quad$ Sí(1). ¿QQuién?

5. Si piensa en la relación con los médicos de su hijo, ¿cómo diría que es?

6. Es frecuente que a los médicos que atienden a niños con enfermedades crónicas desde pequeños, al crecer, les sea difícil dirigirse directamente a los adolescentes para conocer su perspectiva respecto a lo que les está pasando.

¿Usted cree que esto pasa con el equipo de salud que atiende a su hijo?

No(0). $\square \quad$ Sí(1). $\square$ ¿Desde qué edad empezaron a tenerlo más en cuenta?

7. ¿Los médicos le suelen hacer preguntas sobre cómo su hijo se va preparando para manejarse solo con lo referente a su salud?

No(0). $\square \quad$ A veces(1). $\square \quad$ Casi siempre(2). $\square$

¿Cree que su hijo está en un momento adecuado para ser atendido por un médico de adultos?

No(0). $\quad$ Sí(1). $\square$ No sé(2). ¿Por qué?

8. ¿Cuál cree que sería la edad ideal para que su hijo pasara a un servicio de adultos?

9. ¿Cómo se imagina que va a ser el pasaje a un hospital de adultos? ¿En qué momento?

10. ¿Ese momento le genera alguna sensación?

No(0). $\square$ Sí(1). ¿Cuál/es? 
11. ¿Le parece que habrá diferencias entre la atención pediátrica y la atención de adultos? No(0). $\square \quad$ Sí(1). ¿Cuál/es?

12. ¿Qué cosas cree que podrían ayudar en el momento de la transferencia a una institución de adultos? (Puede responder más de una opción).

- Conocer el lugar(1).

- Que esté cerca de su casa(2)..

- Contar con un resumen de historia clínica (3).

- Que conozcan a su hijo cuando llegue(4).

- Médicos formados en la patología de su hijo (5).

- Vía de comunicación con el hospital (6).

13. Desde el equipo médico, ¿ya le han planteado el pasaje a la atención adultos? No(0). $\square \quad$ Sí(1). $\square$

Si ya se planteó: ¿Cómo se sintió cuando le hablaron por primera vez de esto?

14. Luego de que se abordó el tema, ¿hubo algún cambio de su parte en lo que respecta a la dinámica habitual de cuidado de salud de su hijo?

No(0). $\quad$ Sí(1). ¿Cuál?

15. ¿Ha hablado con su hijo sobre su futuro?

No(0). $\square \quad$ Sí(1). $\square$

16. ¿Cómo se imagina que será la vida de su hijo en 5 años?

17. ¿Y en 10 años?

18. ¿Quiere decir algo más? 